\title{
Periodic solution for neutral-type inertial neural networks with time-varying delays
}

Mei $\mathrm{Xu}^{1 *}$ and Bo Du'D

\author{
"Correspondence: \\ xumei93@126.com \\ 'School of Mathematics and \\ Statistics, Huaiyin Normal University, \\ Huaian, Jiangsu, China
}

\begin{abstract}
In this paper the problems of the existence and stability of periodic solutions of neutral-type inertial neural networks with time-varying delays are discussed by applying Mawhin's continuation theorem and Lyapunov functional method. Finally, two numerical examples are given to illustrate our theoretical results.
\end{abstract}

MSC: $34 \mathrm{~B} 15$

Keywords: Periodic solution; Neutral-type; Inertial neural networks; Stability; Existence

\section{Introduction}

In 1997, Wheeler and Schieve [1] introduced inductance into neural networks and gained a second-order system which is called an inertial neural network (INN). Noticing that lots of previous works mainly pay close attention to neural networks with only the first derivative of the states, so it is significantly important to introduce an inertial term. In INNs, inertial terms are described by the first-order derivative terms which have important meaning in engineering technology, biology, physics and information systems, for more details, see, e.g., [1-3]. Since the inertial terms exist in a neural network, it is very difficult to investigate the dynamic properties of the network system. In the past few decades, many researchers have used different methods and techniques to study INNs in depth and obtained many results. In [4], the authors investigated the global dissipativity for INNs with time-varying delays and parameter uncertainties by using generalized Halanay inequality, matrix measure, and matrix-norm inequality. Wang and Jiang [5] considered a class of impulsive INNs with time-varying delays. The global exponential stability in Lagrange sense for INNs with delays has been discussed in [6, 7]. Draye, Winters, and Cheron [8] studied a class of self-selected modular recurrent neural networks with postural and inertial subnetworks.

In general, periodic solutions of network systems have many important applications in the real world. Thus, in the past few decades, periodic solutions of network systems have been widely studied and gained many important results. For example, in [9], existence and global exponential stability of periodic solutions for discrete-time BAM neural networks have been considered. Furthermore, using suitable Lyapunov function and coincidence

(c) The Author(s) 2020. This article is licensed under a Creative Commons Attribution 4.0 International License, which permits use sharing, adaptation, distribution and reproduction in any medium or format, as long as you give appropriate credit to the original author(s) and the source, provide a link to the Creative Commons licence, and indicate if changes were made. The images or other third party material in this article are included in the article's Creative Commons licence, unless indicated otherwise in a credit line to the material. If material is not included in the article's Creative Commons licence and your intended use is not permitted by statutory regulation or exceeds the permitted use, you will need to obtain permission directly from the copyright holder. To view a copy of this licence, visit http://creativecommons.org/licenses/by/4.0/. 
degree theory, Zhou et al. [10] studied a class of BAM neural networks with periodic coefficients and continuously distributed delays. For more results on periodic solutions of network systems, see, e.g., [11-15]. Lu and Chen [16] studied the global stability of nonnegative equilibria for a Cohen-Grossberg neural network system. Ding, Liu, and Nieto [17] obtained existence of positive almost periodic solutions to a class of hematopoiesis models. In very recent years, Hien and Hai-An [18] considered the problems of positive solutions and exponential stability of positive equilibrium of INNs with multiple timevarying delays as follows:

$$
\frac{d^{2} x_{i}(t)}{d t^{2}}=-a_{i} \frac{d x_{i}(t)}{d t}-b_{i} x_{i}(t)+\sum_{j=1}^{n} c_{i j} f_{j}\left(x_{j}(t)\right)+\sum_{j=1}^{n} d_{i j} f_{j}\left(x_{j}\left(t-\tau_{j}(t)\right)\right)+I_{i}(t),
$$

where $t \geq 0, i=1, \ldots, n$. For the meaning of parameters in (1.1), see [18]. Using the comparison principle and homeomorphisms, the authors obtained some dynamic properties of a positive solution of system (1.1).

A neutral-type $\mathrm{NN}$ is a nonlinear system which shows neutral properties by involving derivatives with delays. Neutral-type INNs is not only an extension of nonneutral-type INNs, but also provides more useful models in many fields, including biology, mechanics, economics, electronics, and so on. Only a few investigations on dynamical properties of neutral-type INNs have been reported so far. In a very recent article, Yogambigai et al. [19] considered a neutral-type INN with discrete and distributed time delays as follows:

$$
\begin{aligned}
\frac{d^{2} x_{i}(t)}{d t^{2}}= & -a_{i} \frac{d x_{i}(t)}{d t}-b_{i} x_{i}(t)+\sum_{j=1}^{n} c_{i j} f_{j}\left(x_{j}(t)\right)+\sum_{j=1}^{n} d_{i j} f_{j}\left(x_{j}(t-h)\right) \\
& +\sum_{j=1}^{n} e_{i j} \int_{t-\tau}^{t} f_{j}\left(x_{j}(s)\right) d s+\sum_{j=1}^{n} h_{i j} \frac{d^{2} x_{i}(t-h)}{d t^{2}}+I_{i}(t) .
\end{aligned}
$$

Global Lagrange stability for system (1.2) was obtained by using LMI method. System (1.2) shows the neutral character by the term $\sum_{j=1}^{n} h_{i j} \frac{d^{2} x_{i}(t-h)}{d t^{2}}$. In fact, according to the Hale's theory [20] for neutral functional differential equations, neutral terms are $D$-operator forms, which are different from the neutral terms in (1.2). In the present paper, we will study a class neutral-type INNs with $D$-operator forms.

In this paper, by using Mawhin's continuation theorem, we obtain existence of periodic solutions for neutral-type inertial neural networks. Mawhin's continuation theorem is a powerful tool for studying periodic solution problems, which is different from other methods, such as fixed-point theorem, variational method, Yoshizawa-type theorem, Masseratype theorem, etc. Furthermore, by using Lyapunov functional method, we obtain stability of periodic solutions for neutral-type inertial neural networks; this method is different from other methods, such as linear matrix inequality (LMI) method, Halanay inequality, matrix measure, and matrix-norm inequality, etc. It is noteworthy that we propose a general approach by the properties of neutral-type operator, Mawhin's continuation theorem and Lyapunov functional method which can be used for studying neutral-type inertial neural networks. The major challenges in this paper are as follows: (1) How to deal with neutral operator in neutral-type INNs and how to use the properties of neutral operator? (2) Since neutral-type INNs contain neutral operator and delays, constructing proper Lypunov function becomes very difficult. 
In this paper, we study the periodic solutions problem for a neutral-type INNs with variable delays. Note that the considered system contains both the neutral terms and variable delays that are all dependent on the properties of $D$-operator (neutral operator). The purpose of this paper is to obtain existence and global asymptotic stability results of periodic solutions via topological degree theory and constructing suitable Lyapunov-Krasovskii functional. Two simulation examples are used to demonstrate the usefulness of our theoretical results. The highlights of this paper are threefold:

(1) In this paper, we study a new class of neutral-type INNs with neutral feature described by $D$-operator which is different from the existing models, see, e.g., [19, 21-25].

(2) For constructing suitable Lyapunov-Krasovskii functional, the neutral operator is first taken into consideration in the neutral-type INNs with variable delays and a nonneutral system can be regarded as a special case.

(3) Different from the previous results, we introduce a new unified framework to deal with the construction of Lyapunov-Krasovskii functional for the neutral-type INNs by using properties of the neutral operator and mathematical analysis tools, which may be of special interest. It is noted that our main results are also valid for the case of a nonneutral system.

The following sections are organized as follows: In Sect. 2, we give preliminaries and problem formulation. In Sect. 3, sufficient conditions are established for existence results of system (2.1). The globally asymptotic stability results of the present paper are given in Sect. 4. In Sect. 5, two numerical examples are given to show the feasibility of our results. Finally, some conclusions and discussions are given about this paper.

\section{Preliminaries and problem formulation}

Denote

$$
S=\{1,2, \ldots, n\}, \quad C_{T}=\{x: x \in C(\mathbb{R}, \mathbb{R}), x(t+T) \equiv x(t)\}, \quad C_{T}^{1}=\left\{x: x^{\prime} \in C_{T}\right\} .
$$

Motivated by the above work, we consider a class of neutral-type INNs with time-varying delays as follows:

$$
\begin{aligned}
\frac{d^{2}\left[A_{i} x_{i}(t)\right]}{d t^{2}}= & -a_{i}(t) \frac{d\left[A_{i} x_{i}(t)\right]}{d t}-b_{i}(t) x_{i}(t)+\sum_{j=1}^{n} c_{i j}(t) f_{j}\left(x_{j}(t)\right) \\
& +\sum_{j=1}^{n} d_{i j}(t) f_{j}\left(x_{j}\left(t-\tau_{j}(t)\right)\right)+I_{i}(t),
\end{aligned}
$$

where $t \geq 0, i \in S, A_{i}$ is a difference operator defined by

$$
\left(A_{i} x_{i}\right)(t)=x_{i}(t)-c_{i} x_{i}(t-\gamma)
$$

$\gamma>0$ and $\left|c_{i}\right| \neq 1$ are constants, $x_{i}(t)$ denotes the state of the $i$ th neuron at time $t, a_{i}(t)>0$ is the damping coefficient, $b_{i}(t)>0$ denotes the strength of different neuron at time $t, c_{i j}(t)$ and $d_{i j}(t)$ are the neuron connection weights at time $t, f_{j}(\cdot)$ is the activation function which is a continuous, $\tau_{j}(t)$ is a delay function with $0 \leq \tau_{j} \leq \hat{\tau}, \hat{\tau}$ is a constant, $I_{i}(t)$ is an external 
input of the $i$ th neuron at time $t$. Initial conditions of system (2.1) are given by

$$
\begin{cases}x_{i}(s)=\phi_{i}(s), & s \in(-\mu, 0], i \in S, \\ \left.x_{i}^{\prime}(s)\right)=\psi_{i}(s), & s \in(-\mu, 0], i \in S,\end{cases}
$$

where $\mu=\max \{\gamma, \hat{\tau}\}$.

Remark 2.1 According to Hale's theory [20], a solution $x_{i}(t)(i \in S)$ of system (2.1) is a function $x_{i} \in C(\mathbb{R}, \mathbb{R})$ such that $A_{i} x_{i} \in C(\mathbb{R}, \mathbb{R})$. From Lemma 2.3, we have $\left(A_{i} x_{i}\right)^{\prime \prime}=A_{i} x_{i}^{\prime \prime}$. So the solution $x_{i}(t)(i \in S)$ of system (2.1) must be in $C^{2}(\mathbb{R}, \mathbb{R})$.

Let

$$
y_{i}(t)=\frac{d\left[A_{i} x_{i}(t)\right]}{d t}+\xi_{i}\left(A_{i} x_{i}\right)(t), \quad i \in S,
$$

where $\xi_{i}>0$ is a constant. Then system (2.1) is changed into the following form:

$$
\left\{\begin{aligned}
\left(A_{i} x_{i}\right)^{\prime}(t)=-\xi_{i}\left(A_{i} x_{i}\right)(t)+y_{i}(t), & \\
y_{i}^{\prime}(t)= & -\left(a_{i}(t)-\xi_{i}\right) y_{i}(t)+\left[\left(a_{i}(t)-\xi_{i}\right) \xi_{i}\right]\left(A_{i} x_{i}\right)(t)-b_{i}(t) A_{i}\left[A_{i}^{-1} x_{i}(t)\right] \\
& +\sum_{j=1}^{n} c_{i j}(t) f_{j}\left(A_{j}\left[A_{j}^{-1} x_{j}(t)\right]\right)+\sum_{j=1}^{n} d_{i j}(t) f_{j}\left(A_{j}\left[A_{j}^{-1} x_{j}\left(t-\tau_{j}(t)\right)\right]\right)+I_{i}(t) .
\end{aligned}\right.
$$

Remark 2.2 The existence of $A_{i}^{-1}$ is based on Lemma 2.3.

Remark 2.3 There are many periodic phenomena in nature and society. One of the important trends in the investigations of inertial neural networks is related to the periodic solutions of these systems. Hence, studying periodic solution problems of system (2.1) has important theoretical and practical value.

Remark 2.4 System (2.1) is a neutral-type INN which shows neutral features due to $\left(A_{i} x_{i}\right)(t)=x_{i}(t)-c_{i} x_{i}(t-\gamma)$. When $c_{i}=0$, system (2.1) is a nonneutral-type INN which has been studied by many authors. Hence, system (2.1) is more general than the existing INNs.

Lemma 2.1 ([26]) Assume that $\mathcal{X}$ and $\mathcal{Y}$ are two Banach spaces, and $L: D(L) \subset \mathcal{X} \rightarrow \mathcal{Y}$ is a Fredholm operator with index zero. Furthermore, suppose that $\Omega \subset \mathcal{X}$ is an open bounded set and $N: \bar{\Omega} \rightarrow \mathcal{Y}$ is L-compact on $\bar{\Omega}$. If all the following conditions hold:

(1) $L x \neq \lambda N x, \forall x \in \partial \Omega \cap D(L), \forall \lambda \in(0,1)$,

(2) $N x \notin \operatorname{Im} L, \forall x \in \partial \Omega \cap \operatorname{Ker} L$,

(3) $\operatorname{deg}\{J Q N, \Omega \cap \operatorname{Ker} L, 0\} \neq 0$,

where $J: \operatorname{Im} Q \rightarrow \operatorname{Ker} L$ is an isomorphism. Then equation $L x=N x$ has a solution on $\bar{\Omega} \cap$ $D(L)$.

Lemma 2.2 ([27]) Let $g \in C_{T}, \tau \in C_{T}^{1}$ with $\tau^{\prime}(t)<1 \forall t \in[0, T]$. Then $g(\mu(t)) \in C_{T}$, where $\mu(t)$ is the inverse function of $t-\tau(t)$. 
Lemma 2.3 ([28]) Let $A: C_{T} \rightarrow C_{T},(A x)(t)=x(t)-c x(t-\tau)$, where $\tau>0$ and $c$ are constants, $C_{T}$ is the space of $T$-periodic continuous functions. If $|c| \neq 1$, then operator $A$ has continuous inverse $A^{-1}$ on $C_{T}$, satisfying

$$
\left[A^{-1} f\right](t)= \begin{cases}\sum_{j \geq 0} c^{j} f(t-j \tau), & \text { if }|c|<1, \forall f \in C_{T}, \\ -\sum_{j \geq 1} c^{-j} f(t+j \tau), & \text { if }|c|>1, \forall f \in C_{T},\end{cases}
$$

and

$$
\left|\left(A^{-1} x\right)(t)\right| \leq \frac{\|x\|}{|1-| c||}, \quad \forall x \in C_{T} .
$$

Lemma 2.4 (Bellman inequality) Assume that $f(t)$ is a nonnegative continuous function on $[0, T]$. If there are constants $\delta, k \geq 0$ such that

$$
f(t) \leq \delta+k \int_{0}^{t} f(s) d s, \quad t \in[0, T]
$$

then

$$
f(t) \leq \delta e^{k t}
$$

Throughout the paper, the following assumptions hold:

$\left(H_{1}\right) a_{i}(t), b_{i}(t), c_{i j}(t)$, and $d_{i j}(t)$ are continuous T-periodic functions.

$\left(H_{2}\right)$ There exists a constant $l_{j} \geq 0$ such that

$$
\left|f_{j}(x)\right| \leq l_{j}, j \in S, \forall x \in \mathbb{R} \text {. }
$$

$\left(H_{3}\right)$ There exists a constant $\hat{l}_{j} \geq 0$ such that

$$
\left|f_{j}(x)\right| \leq \hat{l}_{j}|x| j \in S, \forall x \in \mathbb{R}
$$

$\left(H_{4}\right)$ There exists a constant $\tilde{l}_{j} \geq 0$ such that

$$
\left|f_{j}(x)-f_{j}(y)\right| \leq \tilde{l}_{j}|x-y|, j \in S, \forall x, y \in \mathbb{R}
$$

Remark 2.5 For obtaining existence of periodic solutions for neutral-type inertial neural networks, assumption $\left(\mathrm{H}_{1}\right)$ is a important sufficient condition. Assumption $\left(\mathrm{H}_{2}\right)$ is an important condition for estimating the prior bounds of the solution by using Mawhin's continuation theorem. Assumption $\left(\mathrm{H}_{4}\right)$ is the famous Lipschitz condition for $f_{j}(x), j=$ $1,2, \ldots, n$. Assumption $\left(\mathrm{H}_{3}\right)$ is a linear growth condition for $f_{j}(x), j=1,2, \ldots, n$. If $f_{j}(0)=0$, then $\left(\mathrm{H}_{4}\right)$ implies $\left(\mathrm{H}_{3}\right)$.

\section{Existence and uniqueness of a periodic solution}

Let $x(t)=\left(x_{1}(t), \ldots, x_{n}(t)\right)^{\top}, y(t)=\left(y_{1}(t), \ldots, y_{n}(t)\right)^{\top}$. Set

$$
\mathcal{X}=\mathcal{Y}=\left\{w(t)=(x(t), y(t))^{\top} \in C\left(\mathbb{R}, \mathbb{R}^{2 n}\right), w(t+T)=w(t)\right\}
$$


with the norm $\|w\|=\max \left\{|x|_{\infty},|y|_{\infty}\right\}$, where

$$
|f|_{\infty}=\max _{i \in S,}\left|f_{i}\right|_{0}, \quad\left|f_{i}\right|_{0}=\max _{t \in \mathbb{R}}\left|f_{i}(t)\right| \quad \forall f \in \mathbb{R}^{n} .
$$

It is easy to see that $\mathcal{X}$ and $\mathcal{Y}$ are two Banach spaces. Let

$$
\begin{aligned}
& L: D(L) \subset \mathcal{X} \rightarrow \mathcal{X}, \quad(L w)(t)=w^{\prime}(t)=\left(x^{\prime}(t), y^{\prime}(t)\right)^{\top}, \quad t \in \mathbb{R}, \\
& (L w)_{i}(t)=\left(A_{i} x_{i}\right)^{\prime}(t), \quad i \in S, t \in \mathbb{R},
\end{aligned}
$$

and

$$
(L w)_{n+i}(t)=y_{i}^{\prime}(t), \quad i \in S, t \in \mathbb{R} .
$$

Let $N: \mathcal{X} \rightarrow \mathcal{X}$ with

$$
(N w)_{i}(t)=-\xi_{i}\left(A_{i} x_{i}\right)(t)+y_{i}(t), \quad i \in S, t \in \mathbb{R},
$$

and

$$
\begin{aligned}
(N w)_{n+i}(t)= & -\left(a_{i}(t)-\xi_{i}\right) y_{i}(t)+\left[\left(a_{i}(t)-\xi_{i}\right) \xi_{i}\right]\left(A_{i} x_{i}\right)(t)-b_{i}(t) A_{i}\left[A_{i}^{-1} x_{i}(t)\right] \\
& +\sum_{j=1}^{n} c_{i j}(t) f_{j}\left(A_{j}\left[A_{j}^{-1} x_{j}(t)\right]\right)+\sum_{j=1}^{n} d_{i j}(t) f_{j}\left(A_{j}\left[A_{j}^{-1} x_{j}\left(t-\tau_{j}(t)\right)\right]\right) \\
& +I_{i}(t), \quad i \in S, t \in \mathbb{R} .
\end{aligned}
$$

Obviously, $\operatorname{Ker} L=\mathbb{R}^{2 n}, \operatorname{Im} L=\left\{w: w \in \mathcal{X}, \int_{0}^{T} w(s) d s=\mathbf{0}\right\}$ is closed in $\mathcal{Y}, \operatorname{dim} \operatorname{Ker} L=$ condim $\operatorname{Im} L=2 n$. So $L$ is a Fredholm operator with index zero. Let

$$
P: \mathcal{X} \rightarrow \operatorname{Ker} L, \quad Q: \mathcal{Y} \rightarrow \mathcal{Y} / \operatorname{Im} L
$$

be defined by

$$
P x=\frac{1}{T} \int_{0}^{T} w(s) d s, \quad Q y=\frac{1}{T} \int_{0}^{T} y(s) d s,
$$

and let

$$
L_{p}=\left.L\right|_{\mathcal{X} \cap \operatorname{Ker} P}: \mathcal{X} \cap \operatorname{Ker} P \rightarrow \operatorname{Im} L .
$$

Then $L_{p}$ has its right inverse $L_{P}^{-1}$.

Theorem 3.1 Suppose that Assumptions $\left(H_{1}\right)$ and $\left(H_{2}\right)$ hold. Then system (2.1) has at least one T-periodic solution, provided that the following conditions hold:

$$
\begin{aligned}
& 1-T\left(\left|a_{i}\right|_{0}+\xi_{i}\right)>0, \quad i \in S \\
& 1-\frac{\left.\left.|1-| c\right|_{i}\left|\left(\left|a_{i}\right|_{0}+\xi_{i}\right) \xi_{i}+\right| b_{i}\right|_{0}}{\left.|1-| c\right|_{i} \mid\left(1-T\left(\left|a_{i}\right|_{0}+\xi_{i}\right)\right)} \frac{T}{1-T \xi_{i}}>0, \quad i \in S
\end{aligned}
$$




$$
\left|\xi_{i}\left(1-c_{i}\right) m\right| \neq|M| \quad \text { or } \quad\left|\xi_{i}\left(1-c_{i}\right) M\right| \neq|m|, \quad i \in S
$$

where $\xi_{i}>0$ is defined by (2.3), and $m$ and $M$ are defined by (3.14) and (3.15), respectively.

Proof Consider the following operator equation:

$$
L w=\lambda N w, \quad w \in D(L), \lambda \in(0,1)
$$

where $L$ and $N$ are defined by (3.1)-(3.4). Let $\Omega_{1}=\{w: w \in D(L), L w=\lambda N w, \lambda \in(0,1)\}$. Then $\forall x \in \Omega_{1}$, it follows that

$$
\begin{aligned}
\left(A_{i} x_{i}\right)^{\prime}(t) & =\lambda\left[-\xi_{i}\left(A_{i} x_{i}\right)(t)+y_{i}(t)\right], \quad i \in S, t \in \mathbb{R}, \\
y_{i}^{\prime}(t)=\lambda\left[-\left(a_{i}(t)-\xi_{i}\right) y_{i}(t)+\left[\left(a_{i}(t)-\xi_{i}\right) \xi_{i}\right]\left(A_{i} x_{i}\right)(t)-b_{i}(t) A_{i}\left[A_{i}^{-1} x_{i}(t)\right]\right. & \\
+ & \sum_{j=1}^{n} c_{i j}(t) f_{j}\left(A_{j}\left[A_{j}^{-1} x_{j}(t)\right]\right) \\
& \left.+\sum_{j=1}^{n} d_{i j}(t) f_{j}\left(A_{j}\left[A_{j}^{-1} x_{j}\left(t-\tau_{j}(t)\right)\right]\right)+I_{i}(t)\right], \quad i \in S, t \in \mathbb{R} .
\end{aligned}
$$

By (3.8), we have

$$
\begin{aligned}
\left|\left(A_{i} x_{i}\right)(t)\right| & \leq\left(1+\left|c_{i}\right|\right)|\phi|_{\infty}+\int_{0}^{T}\left|\left(A_{i} x_{i}\right)^{\prime}(t)\right| d t \\
& \leq\left(1+\left|c_{i}\right|\right)|\phi|_{\infty}+\xi_{i} \int_{0}^{T}\left|\left(A_{i} x_{i}\right)(t)\right| d t+\int_{0}^{T}\left|y_{i}(t)\right| d t .
\end{aligned}
$$

By the above inequality, we have

$$
\left|A_{i} x_{i}\right|_{0}=\max _{t \in[0, T]}\left|A_{i} x_{i}(t)\right| \leq\left(1+\left|c_{i}\right|\right)|\phi|_{\infty}+\xi_{i} T\left|\left(A_{i} x_{i}\right)\right|_{0}+T\left|y_{i}\right|_{0}
$$

Using condition (3.5) and the above inequality, we then get

$$
\left|A_{i} x_{i}\right|_{0} \leq \frac{\left(1+\left|c_{i}\right|\right)|\phi|_{\infty}}{1-T \xi_{i}}+\frac{T\left|y_{i}\right|_{0}}{1-T \xi_{i}}
$$

From Assumption $\left(\mathrm{H}_{2}\right)$, (3.10), and Lemma 2.3, we get

$$
\begin{aligned}
\left|y_{i}(t)\right| \leq & |\tilde{\psi}|_{\infty}+\int_{0}^{T}\left|y_{i}^{\prime}(t)\right| d t \\
\leq & |\tilde{\psi}|_{\infty}+\int_{0}^{T}\left(\left|a_{i}\right|_{0}+\xi_{i}\right)\left|y_{i}(t)\right| d t \\
& +\left(\left|a_{i}\right|_{0}+\xi_{i}\right) \xi_{i} \int_{0}^{T}\left|\left(A_{i} x_{i}\right)(t)\right| d t+\left|b_{i}\right|_{0} \int_{0}^{T}\left|x_{i}(t)\right| d t \\
& +\sum_{j=1}^{n}\left(\left|c_{i j}\right|_{0}+\left|d_{i j}\right|_{0}\right) l_{j} T+T\left|I_{i}\right|_{0}
\end{aligned}
$$




$$
\begin{aligned}
& \leq|\tilde{\psi}|_{\infty}+T\left(\left|a_{i}\right|_{0}+\xi_{i}\right)\left|y_{i}\right|_{0} \\
& \quad+\left(\left|a_{i}\right|_{0}+\xi_{i}\right) \xi_{i} \int_{0}^{T}\left|\left(A_{i} x_{i}\right)(t)\right| d t+\frac{\left|b_{i}\right|_{0}}{\left.|1-| c\right|_{i} \mid} \int_{0}^{T}\left|\left(A_{i} x_{i}\right)(t)\right| d t \\
& \quad+\sum_{j=1}^{n}\left(\left|c_{i j}\right|_{0}+\left|d_{i j}\right|_{0}\right) l_{j} T+T\left|I_{i}\right|_{0} \\
& =|\tilde{\psi}|_{\infty}+\sum_{j=1}^{n}\left(\left|c_{i j}\right|_{0}+\left|d_{i j}\right|_{0}\right) l_{j} T+T\left|I_{i}\right|_{0}+T\left(\left|a_{i}\right|_{0}+\xi_{i}\right)\left|y_{i}\right|_{0} \\
& \quad+\frac{\left.\left.|1-| c\right|_{i}\left|\left(\left|a_{i}\right|_{0}+\xi_{i}\right) \xi_{i}+\right| b_{i}\right|_{0}}{\left.|1-| c\right|_{i} \mid} \int_{0}^{T}\left|\left(A_{i} x_{i}\right)(t)\right| d t,
\end{aligned}
$$

where $|\tilde{\psi}|_{\infty}=\max _{i \in S}\left\{\left|A_{i} \psi(0)+\xi_{i} \phi(0)\right|\right\}$. Due to condition (3.5),

$$
\begin{aligned}
\left|y_{i}\right|_{0} \leq & \frac{|\tilde{\psi}|_{\infty}+\sum_{j=1}^{n}\left(\left|c_{i j}\right|_{0}+\left|d_{i j}\right|_{0}\right) l_{j} T+T\left|I_{i}\right|_{0}}{1-T\left(\left|a_{i}\right|_{0}+\xi_{i}\right)} \\
& +\frac{\left.\left.|1-| c\right|_{i}\left|\left(\left|a_{i}\right|_{0}+\xi_{i}\right) \xi_{i}+\right| b_{i}\right|_{0}}{\left.|1-| c\right|_{i} \mid\left(1-T\left(\left|a_{i}\right|_{0}+\xi_{i}\right)\right)} \int_{0}^{T}\left|\left(A_{i} x_{i}\right)(t)\right| d t .
\end{aligned}
$$

In view of (3.10), we have

$$
\begin{aligned}
\left|y_{i}\right|_{0} \leq & \frac{|\tilde{\psi}|_{\infty}+\sum_{j=1}^{n}\left(\left|c_{i j}\right|_{0}+\left|d_{i j}\right|_{0}\right) l_{j} T+T\left|I_{i}\right|_{0}}{1-T\left(\left|a_{i}\right|_{0}+\xi_{i}\right)} \\
& +\frac{\left.\left.|1-| c\right|_{i}\left|\left(\left|a_{i}\right|_{0}+\xi_{i}\right) \xi_{i}+\right| b_{i}\right|_{0}}{\left.|1-| c\right|_{i} \mid\left(1-T\left(\left|a_{i}\right|_{0}+\xi_{i}\right)\right)} \frac{\left(1+\left|c_{i}\right|\right)|\phi|_{\infty}}{1-T \xi_{i}} \\
& +\frac{\left.\left.|1-| c\right|_{i}\left|\left(\left|a_{i}\right|_{0}+\xi_{i}\right) \xi_{i}+\right| b_{i}\right|_{0}}{\left.|1-| c\right|_{i} \mid\left(1-T\left(\left|a_{i}\right|_{0}+\xi_{i}\right)\right)} \frac{T\left|y_{i}\right|_{0}}{1-T \xi_{i}} .
\end{aligned}
$$

From condition (3.6), there exists a positive constant $K_{i}$ such that

$$
\left|y_{i}\right|_{0} \leq K_{i}, \quad i \in S, \forall t \in[0, T]
$$

Using (3.10) and (3.11), we obtain

$$
\left|A_{i} x_{i}\right|_{0} \leq \frac{\left(1+\left|c_{i}\right|\right)|\phi|_{\infty}}{1-T \xi_{i}}+\frac{T K_{i}}{1-T \xi_{i}}
$$

In view of Lemma 2.3,

$$
\begin{aligned}
\left|x_{i}\right|_{0} & \leq \frac{\left|A_{i} x_{i}\right|_{0}}{|1-| c_{i}||} \\
& \leq \frac{\left(1+\left|c_{i}\right|\right)|\phi|_{\infty}}{|1-| c_{i}||\left(1-T \xi_{i}\right)}+\frac{T K_{i}}{|1-| c_{i}||\left(1-T \xi_{i}\right)} \\
& :=P_{i} .
\end{aligned}
$$

From (3.11) and (3.12), we have

$$
\|w\|=\max \left\{\max _{i \in S} K_{i}, \max _{i \in S} P_{i}\right\}:=M .
$$


Let $\Omega_{2}=\{w \in \mathcal{X}:\|w\|<M+1\}$. Then $\forall w \in \Omega_{2}$, condition (1) of Lemma 2.1 holds. We prove that

$$
Q N w \neq \mathbf{0} \quad \forall w \in \partial \Omega_{2} \cap \operatorname{Ker} L .
$$

In fact, $\forall w \in \partial \Omega_{2} \cap \operatorname{Ker} L$, then $w \in \mathbb{R}^{2 n}$ is a constant vector, and there exists at least one $i \in S$ such that

$$
\left|y_{i}\right|=M+1 \quad \text { and } \quad\left|x_{i}\right|=m<M+1 .
$$

If $y_{i}=M+1, x_{i}=m$, integrate (3.3) over [0,T], then $\xi_{i}\left(1-c_{i}\right) m=M$, which is a contradiction to (3.7). If $y_{i}=-(M+1), x_{i}= \pm m$, we can obtain a similar contradiction to (3.7). On the other hand, if there exists at least one $i \in S$ such that

$$
\left|x_{i}\right|=M+1 \quad \text { and } \quad\left|y_{i}\right|=m<M+1
$$

then we can obtain similar results. Hence, (3.13) and condition (2) of Lemma 2.1 hold. Let

$$
H_{i}\left(w_{i}, \mu\right)=\mu w_{i}+(1-\mu) Q N w_{i}, \quad \mu \in[0,1], i=1,2, \ldots, 2 n .
$$

Using (3.13), we have

$$
H_{i}\left(w_{i}, \mu\right) \neq 0 \neq 0 \quad \text { for all } w \in \partial \Omega_{2} \cap \operatorname{Ker} L, i=1,2, \ldots, 2 n \text {. }
$$

Based on the property of topological degree and taking $J$ to be the identity mapping $I$ : $\operatorname{Im} Q \rightarrow \operatorname{Ker} L$, then

$$
\begin{aligned}
\operatorname{deg}\left\{U Q N, \Omega_{2} \cap \operatorname{Ker} L, 0\right\} & =\operatorname{deg}\left\{H(\cdot, 0), \Omega_{2} \cap \operatorname{Ker} L, 0\right\} \\
& =\operatorname{deg}\left\{H(\cdot, 1), \Omega_{2} \cap \operatorname{Ker} L, 0\right\} \\
& =1 \neq 0 .
\end{aligned}
$$

So, condition (3) of Lemma 2.1 holds. Therefore, by using Lemma 2.1, we see that the equation $L x=N x$ has at least one $T$-periodic solution $w$ in $\bar{\Omega}_{2}$. Namely, system (2.1) has at least one positive $T$-periodic solution.

Theorem 3.2 Suppose that $\tau_{j}^{\prime}(t)<1(j \in S, t \in \mathbb{R})$, and Assumptions $\left(H_{1}\right)$ and $\left(H_{3}\right)$ hold. Then system (2.1) has at least one T-periodic solution, provided that the following conditions hold:

$$
\begin{aligned}
& 1-T\left(\left|a_{i}\right|_{0}+\xi_{i}\right)>0, \quad i \in S, \\
& 1-\frac{T^{2}|1-| c_{i}||\left(\left|a_{i}\right|_{0}+\xi_{i}\right) \xi_{i}+T^{2}\left|b_{i}\right|_{0}+\left|\Gamma_{i j}\right|_{0}}{|1-| c_{i}||\left(1-T\left(\left|a_{i}\right|_{0}+\xi_{i}\right)\right)\left(1-T \xi_{i}\right)}>0, \quad i, j \in S, \\
& \left|\xi_{i}\left(1-c_{i}\right) m\right| \neq|M| \quad \text { or } \quad\left|\xi_{i}\left(1-c_{i}\right) M\right| \neq|m|, \quad i \in S,
\end{aligned}
$$

where $\xi_{i}>0$ is defined by (2.3), and $M$ is defined by (3.27), $m \leq M+1$ is a positive constant, $\Gamma_{i j}(t)=c_{i j}(t)+\frac{d_{i j}\left(\mu_{j}(t)\right)}{1-\tau^{\prime}\left(\mu_{j}(t)\right)}, \mu_{j}(t)$ is a inverse function of $t-\tau_{j}(t)$. 
Proof We only show that the solutions to system (3.8) and (3.9) are bounded, other proofs are similar to that of Theorem 3.1. By (3.10) and Lemma 2.3, we get

$$
\begin{aligned}
\left|y_{i}(t)\right| \leq & |\tilde{\psi}|_{\infty}+\int_{0}^{T}\left|y_{i}^{\prime}(t)\right| d t \\
\leq & |\tilde{\psi}|_{\infty}+\int_{0}^{T}\left(\left|a_{i}\right|_{0}+\xi_{i}\right)\left|y_{i}(t)\right| d t \\
& +\left(\left|a_{i}\right|_{0}+\xi_{i}\right) \xi_{i} \int_{0}^{T}\left|\left(A_{i} x_{i}\right)(t)\right| d t+\left|b_{i}\right|_{0} \int_{0}^{T}\left|x_{i}(t)\right| d t \\
& +\sum_{j=1}^{n} \int_{0}^{T}\left|c_{i j}(s) f_{j}\left(x_{j}(s)\right)\right| d s+\sum_{j=1}^{n} \int_{0}^{T}\left|d_{i j}(s) f_{j}\left(x_{j}\left(s-\tau_{j}(s)\right)\right)\right| d s+T\left|I_{i}\right|_{0} .
\end{aligned}
$$

Consider the term $\int_{0}^{T} \sum_{j=1}^{n} d_{i j}(s) f_{j}\left(x_{j}\left(s-\tau_{j}(s)\right)\right) d s$ in (3.19). Using Lemma 2.2, we have

$$
\int_{0}^{T} \sum_{j=1}^{n}\left|d_{i j}(s) f_{j}\left(x_{j}\left(s-\tau_{j}(s)\right)\right)\right| d s=\int_{0}^{T} \sum_{j=1}^{n} \frac{\left|d_{i j}\left(\mu_{j}(s)\right)\right|}{1-\tau^{\prime}\left(\mu_{j}(s)\right)}\left|f_{j}\left(x_{j}(s)\right)\right| d s, \quad i \in S,
$$

where $\mu_{j}(t)$ is a inverse function of $t-\tau_{j}(t)$. From Assumption $\left(\mathrm{H}_{3}\right),(3.19)$, and (3.20), we have

$$
\begin{aligned}
\left|y_{i}(t)\right| \leq & |\tilde{\psi}|_{\infty}+\int_{0}^{T}\left|y_{i}^{\prime}(t)\right| d t \\
\leq & |\tilde{\psi}|_{\infty}+T\left(\left|a_{i}\right|_{0}+\xi_{i}\right)\left|y_{i}\right|_{0} \\
& +\left(\left|a_{i}\right|_{0}+\xi_{i}\right) \xi_{i} \int_{0}^{T}\left|\left(A_{i} x_{i}\right)(t)\right| d t+\frac{\left|b_{i}\right|_{0}}{\left.|1-| c\right|_{i} \mid} \int_{0}^{T}\left|\left(A_{i} x_{i}\right)(t)\right| d t \\
& +\int_{0}^{T} \sum_{j=1}^{n}\left|\Gamma_{i j}(s) f_{j}\left(x_{j}(s)\right)\right| d s+T\left|I_{i}\right|_{0} \\
\leq & |\tilde{\psi}|_{\infty}+T\left(\left|a_{i}\right|_{0}+\xi_{i}\right)\left|y_{i}\right|_{0} \\
& +\left(\left|a_{i}\right|_{0}+\xi_{i}\right) \xi_{i} \int_{0}^{T}\left|\left(A_{i} x_{i}\right)(t)\right| d t+\frac{\left|b_{i}\right|_{0}}{\left.|1-| c\right|_{i} \mid} \int_{0}^{T}\left|\left(A_{i} x_{i}\right)(t)\right| d t \\
& +\left.T \hat{l}_{j}\left|\sum_{j=1}^{n}\right| \Gamma_{i j}\right|_{0}|x|_{\infty}+T\left|I_{i}\right|_{0} .
\end{aligned}
$$

Due to condition (3.16), then

$$
\begin{aligned}
\left|y_{i}\right|_{0} \leq & \frac{|\tilde{\psi}|_{\infty}+T\left|I_{i}\right|_{0}}{1-T\left(\left|a_{i}\right|_{0}+\xi_{i}\right)} \\
& +\frac{\left.\left.|1-| c\right|_{i}\left|\left(\left|a_{i}\right|_{0}+\xi_{i}\right) \xi_{i}+\right| b_{i}\right|_{0}}{\left.|1-| c\right|_{i} \mid\left(1-T\left(\left|a_{i}\right|_{0}+\xi_{i}\right)\right)} T\left|A_{i} x_{i}\right|_{0} \\
& +\frac{\sum_{j=1}^{n}\left|\Gamma_{i j}\right|_{0}}{1-T\left(\left|a_{i}\right|_{0}+\xi_{i}\right)}|x|_{\infty} .
\end{aligned}
$$


By (3.10) and (3.21), then

$$
\begin{aligned}
\left|y_{i}\right|_{0} \leq & \frac{|\tilde{\psi}|_{\infty}+T\left|I_{i}\right|_{0}}{1-T\left(\left|a_{i}\right|_{0}+\xi_{i}\right)} \\
& +\frac{|1-| c_{i}||\left(\left|a_{i}\right|_{0}+\xi_{i}\right) \xi_{i}+\left|b_{i}\right|_{0}}{|1-| c_{i}||\left(1-T\left(\left|a_{i}\right|_{0}+\xi_{i}\right)\right)} \frac{T\left(1+\left|c_{i}\right|\right)|\phi|_{\infty}}{1-T \xi_{i}} \\
& +\frac{|1-| c_{i}||\left(\left|a_{i}\right|_{0}+\xi_{i}\right) \xi_{i}+\left|b_{i}\right|_{0}}{|1-| c_{i}||\left(1-T\left(\left|a_{i}\right|_{0}+\xi_{i}\right)\right)} \frac{T^{2}\left|y_{i}\right|_{0}}{1-T \xi_{i}} \\
& +\frac{\sum_{j=1}^{n}\left|\Gamma_{i j}\right|_{0}}{1-T\left(\left|a_{i}\right|_{0}+\xi_{i}\right)}|x|_{\infty} .
\end{aligned}
$$

From Lemma 2.3 and (3.10), we get

$$
|x|_{\infty} \leq \frac{\left(1+\left|c_{i}\right|\right)|\phi|_{\infty}}{|1-| c_{i}||\left(1-T \xi_{i}\right)}+\frac{T|y|_{\infty}}{|1-| c_{i}||\left(1-T \xi_{i}\right)}
$$

By (3.22) and (3.23), we have

$$
\begin{aligned}
|y|_{\infty} \leq & \frac{|\tilde{\psi}|_{\infty}+T\left|I_{i}\right|_{0}}{1-T\left(\left|a_{i}\right|_{0}+\xi_{i}\right)} \\
& +\frac{|1-| c_{i}||\left(\left|a_{i}\right|_{0}+\xi_{i}\right) \xi_{i}+\left|b_{i}\right|_{0}}{|1-| c_{i}||\left(1-T\left(\left|a_{i}\right|_{0}+\xi_{i}\right)\right)} \frac{T\left(1+\left|c_{i}\right|\right)|\phi|_{\infty}}{1-T \xi_{i}} \\
& +\frac{|1-| c_{i}||\left(\left|a_{i}\right|_{0}+\xi_{i}\right) \xi_{i}+\left|b_{i}\right|_{0}}{|1-| c_{i}||\left(1-T\left(\left|a_{i}\right|_{0}+\xi_{i}\right)\right)} \frac{T^{2}|y|_{\infty}}{1-T \xi_{i}} \\
& +\frac{\sum_{j=1}^{n}\left|\Gamma_{i j}\right|_{0}}{1-T\left(\left|a_{i}\right|_{0}+\xi_{i}\right)} \frac{\left(1+\left|c_{i}\right|\right)|\phi|_{\infty}}{|1-| c_{i}||\left(1-T \xi_{i}\right)} \\
& +\frac{\sum_{j=1}^{n}\left|\Gamma_{i j}\right|_{0}}{1-T\left(\left|a_{i}\right|_{0}+\xi_{i}\right)} \frac{T|y|_{\infty}}{|1-| c_{i}||\left(1-T \xi_{i}\right)} .
\end{aligned}
$$

From condition (3.17) and (3.24), there exists a constant $M_{1}>0$ such that

$$
|y|_{\infty} \leq M_{1}
$$

In view of (3.23) and (3.25), there exists a constant $M_{2}>0$ such that

$$
|x|_{\infty} \leq M_{2}
$$

It follows from (3.25) and (3.26), there there exists a constant $M>0$ such that

$$
\|\omega\|=\max \left\{|x|_{\infty},|y|_{\infty}\right\} \leq M
$$

The following proof is similar to the corresponding arguments in the proof of Theorem 3.1, so we omit it.

Due to Assumption $\left(\mathrm{H}_{4}\right)$, the term $f_{j}\left(x_{j}\right), j \in S$ in system (2.1) satisfies Lipschitz condition on $\mathbb{R}$. Thus, by basic results for functional differential equations, we have the following theorems for the unique existence of a periodic solution to system (2.1). 
Theorem 3.3 Suppose all the conditions of Theorem 3.1 and Assumption $\left(H_{4}\right)$ hold. Then system (2.1) has a unique T-periodic solution.

Proof Assume that $w(t)=(x(t), y(t))^{\top}$ and $\kappa(t)=(u(t), v(t))^{\top}$ are two periodic solutions of system (2.3) which satisfy the initial conditions

$$
\begin{cases}x_{i}(s)=\phi_{i}(s), & s \in(-\mu, 0], i \in S, \\ \left.x_{i}^{\prime}(s)\right)=\psi_{i}(s), & s \in(-\mu, 0], i \in S .\end{cases}
$$

Then, we have

$$
\left|A_{i} x_{i}(t)-A_{i} u_{i}(t)\right| \leq \int_{0}^{t}\left(\xi_{i}\left|A_{i} x_{i}(s)-A_{i} u_{i}(s)\right|+\left|y_{i}(s)-v_{i}(s)\right|\right) d s .
$$

By (3.28), we get

$$
\sum_{i=1}^{n}\left|A_{i} x_{i}(t)-A_{i} u_{i}(t)\right| \leq \int_{0}^{t} \sum_{i=1}^{n} \xi_{i}\left|A_{i} x_{i}(s)-A_{i} u_{i}(s)\right| d s+T \sum_{i=1}^{n}\left|y_{i}(t)-v_{i}(t)\right|_{0} .
$$

By assumptions of Theorem 3.3, we have

$$
\begin{aligned}
\mid y_{i}(t) & -v_{i}(t) \mid \\
\leq & \int_{0}^{t}\left|a_{i}(t)-\xi_{i}\right|_{0}\left|y_{i}(s)-v_{i}(s)\right| d s \\
& +\left|\left(a_{i}(t)-\xi_{i}\right) \xi_{i}\right|_{0} \int_{0}^{t}\left|A_{i} x_{i}(s)-A_{i} u_{i}(s)\right| d s+\left|b_{i}\right|_{0} \int_{0}^{t}\left|x_{i}(s)-u_{i}(s)\right| d s \\
& +\int_{0}^{t} \sum_{j=1}^{n}\left(\left|c_{i j}\right|_{0}+\left|d_{i j}\right|_{0}\right) \tilde{l}_{j}\left|x_{j}(s)-u_{j}(s)\right| d s \\
\leq & \int_{0}^{t}\left|a_{i}(t)-\xi_{i}\right|_{0}\left|y_{i}(s)-v_{i}(s)\right| d s+\left|\left(a_{i}(t)-\xi_{i}\right) \xi_{i}\right|_{0} \int_{0}^{t}\left|A_{i} x_{i}(s)-A_{i} u_{i}(s)\right| d s \\
& +\frac{\left|b_{i}\right|_{0}}{|1-| c_{i}||} \int_{0}^{t}\left|A_{i} x_{i}(s)-A_{i} u_{i}(s)\right| d s \\
& +\int_{0}^{t} \sum_{j=1}^{n} \vartheta_{1}\left|A_{j} x_{j}(s)-A_{j} u_{j}(s)\right| d s,
\end{aligned}
$$

where $\vartheta_{1}=\max _{i, j \in S} \frac{\left(\left|c_{i j}\right| 0+\left|d_{i j}\right| 0 \tilde{l}_{j}\right.}{|1-| c_{i}||}$. By (3.30), we get

$$
\begin{aligned}
\vartheta_{2} \sum_{i=1}^{n}\left|y_{i}(t)-v_{i}(t)\right|_{0} \leq & \sum_{i=1}^{n}\left|\left(a_{i}(t)-\xi_{i}\right) \xi_{i}\right|_{0} \int_{0}^{t}\left|A_{i} x_{i}(s)-A_{i} u_{i}(s)\right| d s \\
& +\sum_{i=1}^{n} \frac{\left|b_{i}\right|_{0}}{|1-| c_{i}||} \int_{0}^{t}\left|A_{i} x_{i}(s)-A_{i} u_{i}(s)\right| d s \\
& +n \vartheta_{1} \int_{0}^{t} \sum_{i=1}^{n}\left|A_{i} x_{i}(s)-A_{i} u_{i}(s)\right| d s,
\end{aligned}
$$


where $\vartheta_{2}=\min _{i \in S}\left(1-T\left|a_{i}(t)-\xi_{i}\right|_{0}\right)>0$. By (3.29) and (3.31), we have

$$
\begin{aligned}
\sum_{i=1}^{n}\left|A_{i} x_{i}(t)-A_{i} u_{i}(t)\right| \leq & \int_{0}^{t} \sum_{i=1}^{n} \xi_{i}\left|A_{i} x_{i}(s)-A_{i} u_{i}(s)\right| d s \\
& \times \frac{T}{\vartheta_{2}} \sum_{i=1}^{n}\left|\left(a_{i}(t)-\xi_{i}\right) \xi_{i}\right|_{0} \int_{0}^{t}\left|A_{i} x_{i}(s)-A_{i} u_{i}(s)\right| d s \\
& +\frac{T}{\vartheta_{2}} \sum_{i=1}^{n} \frac{\left|b_{i}\right|_{0}}{|1-| c_{i}||} \int_{0}^{t}\left|A_{i} x_{i}(s)-A_{i} u_{i}(s)\right| d s \\
& +\frac{T n}{\vartheta_{2}} \vartheta_{1} \int_{0}^{t} \sum_{i=1}^{n}\left|A_{i} x_{i}(s)-A_{i} u_{i}(s)\right| d s .
\end{aligned}
$$

By Lemma 2.4 (Bellman inequality) and (3.32), we have

$$
\sum_{i=1}^{n}\left|A_{i} x_{i}(t)-A_{i} u_{i}(t)\right|=0
$$

i.e.,

$$
x_{i}(t)=u_{i}(t), \quad i \in S .
$$

By (3.31) and (3.33), we have

$$
y_{i}(t)=v_{i}(t), \quad i \in S .
$$

Hence, the periodic solution of system (2.3) is unique, i.e., the periodic solution of system (2.1) is unique.

Theorem 3.4 Suppose all the conditions of Theorem 3.2 and Assumption $\left(H_{4}\right)$ hold. Then system (2.1) has a unique T-periodic solution.

Proof The proof of Theorem 3.4 is similar to that of Theorem 3.3, so we omit it.

\section{Asymptotic behavior of a periodic solution}

Since system (2.3) is equivalent to system (2.1) under the transformation (2.2), we will consider the asymptotic stability problems of system (2.3).

Definition 4.1 If $w^{*}(t)=\left(x_{1}^{*}(t), \ldots, x_{n}^{*}(t), y_{1}^{*}(t), \ldots, y_{n}^{*}(t)\right)^{\top}$ is a periodic solution of system (2.3) and $w(t)=\left(x_{1}(t), \ldots, x_{n}(t), y_{1}(t), \ldots, y_{n}(t)\right)^{\top}$ is any solution of system (2.3) satisfying

$$
\lim _{t \rightarrow+\infty} \sum_{i=1}^{n}\left[\left|x_{i}(t)-x_{i}^{*}(t)\right|+\left|y_{i}(t)-y_{i}^{*}(t)\right|\right]=0 .
$$

Then $w^{*}(t)$ is globally asymptotically stable. 
Theorem 4.1 Under the conditions of Theorem 3.3, assume further that there exist $\iota_{i}>0, \kappa_{i}>0$ such that

$$
\iota_{i}=\lim _{t \rightarrow+\infty} \inf \left[2 \xi_{i}-1-\left(a_{i}(t)-\xi_{i}\right)^{2} \xi_{i}^{2}-\frac{b_{i}(t)}{|1-| c_{i}||^{2}}\right], \quad i \in S
$$

and

$$
\kappa_{i}=\lim _{t \rightarrow+\infty} \inf \left[2\left(a_{i}(t)-\xi_{i}\right)-2-b_{i}(t)-4 \sum_{j=1}^{n}\left(\left|c_{i j}(t)\right|+\left|d_{i j}(t)\right|\right) l_{j}\right], \quad i \in S
$$

where $2\left(a_{i}(t)-\xi_{i}\right)-2-b_{i}(t)>0$ for $t \in \mathbb{R}, i \in S$. Then system (2.3) has a unique T-periodic solution $w^{*}(t)=\left(x_{1}^{*}(t), \ldots, x_{n}^{*}(t), y_{1}^{*}(t), \ldots, y_{n}^{*}(t)\right)^{\top}$ which is globally asymptotically stable.

Proof By Theorem 3.3, system (2.3) has a unique $T$-periodic solution $w^{*}(t)$. Suppose $w(t)$ is any solution of system (2.3). Let

$$
V_{i}(t)=\left(A_{i} x_{i}(t)-A_{i} x_{i}^{*}\right)^{2}+\left(y_{i}(t)-y_{i}^{*}\right)^{2}, \quad i \in S, t \geq 0
$$

Taking the derivative of (4.3) along the solution of (2.3) gives

$$
\begin{aligned}
V_{i}^{\prime}(t)= & 2\left(A_{i} x_{i}(t)-A_{i} x_{i}^{*}\right)\left[A_{i} x_{i}^{\prime}(t)-A_{i} x_{i}^{*^{\prime}}(t)\right]+2\left(y_{i}(t)-y_{i}^{*}\right)\left(y_{i}^{\prime}(t)-y_{i}^{*^{\prime}}(t)\right) \\
= & 2\left(A_{i} x_{i}(t)-A_{i} x_{i}^{*}\right)\left[-\xi_{i}\left(A_{i} x_{i}\right)(t)+y_{i}(t)-\left(-\xi_{i}\left(A_{i} x_{i}^{*}\right)(t)+y_{i}^{*}(t)\right)\right] \\
& +2\left(y_{i}(t)-y_{i}^{*}\right)\left[-\left(a_{i}(t)-\xi_{i}\right) y_{i}(t)+\left[\left(a_{i}(t)-\xi_{i}\right) \xi_{i}\right]\left(A_{i} x_{i}\right)(t)-b_{i}(t) x_{i}(t)\right. \\
& +\sum_{j=1}^{n} c_{i j}(t) f_{j}\left(x_{j}(t)\right)+\sum_{j=1}^{n} d_{i j}(t) f_{j}\left(x_{j}\left(t-\tau_{j}(t)\right)\right)+I_{i}(t) \\
& -\left(-\left(a_{i}(t)-\xi_{i}\right) y_{i}^{*}(t)+\left[\left(a_{i}(t)-\xi_{i}\right) \xi_{i}^{*}\right]\left(A_{i} x_{i}^{*}\right)(t)-b_{i}(t) x_{i}^{*}(t)\right. \\
& \left.\left.+\sum_{j=1}^{n} c_{i j}(t) f_{j}\left(x_{j}^{*}(t)\right)+\sum_{j=1}^{n} d_{i j}(t) f_{j}\left(x_{j}^{*}\left(t-\tau_{j}(t)\right)\right)+I_{i}(t)\right)\right] \\
= & -2 \xi_{i}\left(A_{i} x_{i}(t)-A_{i} x_{i}^{*}\right)^{2}+2\left(A_{i} x_{i}(t)-A_{i} x_{i}^{*}\right)\left(y_{i}(t)-y_{i}^{*}\right) \\
& -2\left(a_{i}(t)-\xi_{i}\right)\left(y_{i}(t)-y_{i}^{*}\right)^{2} \\
& +2\left[\left(a_{i}(t)-\xi_{i}\right) \xi_{i}\right]\left(A_{i} x_{i}(t)-A_{i} x_{i}^{*}\right)\left(y_{i}(t)-y_{i}^{*}\right) \\
& -2 b_{i}(t)\left(x_{i}(t)-x_{i}^{*}\right)\left(y_{i}(t)-y_{i}^{*}\right) \\
& +2\left(y_{i}(t)-y_{i}^{*}\right) \sum_{j=1}^{n} c_{i j}(t)\left[f_{j}\left(x_{j}(t)\right)-f_{j}\left(x_{j}^{*}(t)\right)\right] \\
\leq & -2 \xi_{i}\left(A_{i} x_{i}(t)-A_{i} x_{i}^{*}\right)^{2}+\left(A_{i} x_{i}(t)-A_{i} x_{i}^{*}\right)^{2}+\left(y_{i}(t)-y_{i}^{*}\right)^{2} \\
& +2\left(y_{i}(t)-y_{i}^{*}\right) \sum_{j=1}^{n} d_{i j}(t)\left[f_{j}\left(x_{j}\left(t-\tau_{j}(t)\right)\right)-f_{j}\left(x_{j}^{*}\left(t-\tau_{j}(t)\right)\right)\right] \\
&
\end{aligned}
$$




$$
\begin{aligned}
& -2\left(a_{i}(t)-\xi_{i}\right)\left(y_{i}(t)-y_{i}^{*}\right)^{2} \\
& +\left[\left(a_{i}(t)-\xi_{i}\right) \xi_{i}\right]^{2}\left(A_{i} x_{i}(t)-A_{i} x_{i}^{*}\right)^{2}+\left(y_{i}(t)-y_{i}^{*}\right)^{2} \\
& +b_{i}(t)\left(x_{i}(t)-x_{i}^{*}\right)^{2}+b_{i}(t)\left(y_{i}(t)-y_{i}^{*}\right)^{2} \\
& +4 \sum_{j=1}^{n}\left(\left|c_{i j}(t)\right|+\left|d_{i j}(t)\right|\right) l_{j}\left|y_{i}(t)-y_{i}^{*}\right| \\
\leq & -2 \xi_{i}\left(A_{i} x_{i}(t)-A_{i} x_{i}^{*}\right)^{2}+\left(A_{i} x_{i}(t)-A_{i} x_{i}^{*}\right)^{2}+\left(y_{i}(t)-y_{i}^{*}\right)^{2} \\
& -2\left(a_{i}(t)-\xi_{i}\right)\left(y_{i}(t)-y_{i}^{*}\right)^{2} \\
& +\left[\left(a_{i}(t)-\xi_{i}\right) \xi_{i}\right]^{2}\left(A_{i} x_{i}(t)-A_{i} x_{i}^{*}\right)^{2}+\left(y_{i}(t)-y_{i}^{*}\right)^{2} \\
& +\frac{b_{i}(t)}{|1-| c_{i}||^{2}}\left(A_{i} x_{i}(t)-A_{i} x_{i}^{*}\right)^{2}+b_{i}(t)\left(y_{i}(t)-y_{i}^{*}\right)^{2} \\
& +4 \sum_{j=1}^{n}\left(\left|c_{i j}(t)\right|+\left|d_{i j}(t)\right|\right) l_{j}\left|y_{i}(t)-y_{i}^{*}\right| \\
= & -\hat{a}_{i}\left(A_{i} x_{i}(t)-A_{i} x_{i}^{*}\right)^{2}-\hat{b}_{i}\left(y_{i}(t)-y_{i}^{*}\right)^{2}+\hat{c}_{i}\left|y_{i}(t)-y_{i}^{*}\right|,
\end{aligned}
$$

where

$$
\begin{aligned}
& \hat{a}_{i}=2 \xi_{i}-1-\left(a_{i}(t)-\xi_{i}\right)^{2} \xi_{i}^{2}-\frac{b_{i}(t)}{|1-| c_{i}||^{2}}, \quad \hat{b}_{i}=2\left(a_{i}(t)-\xi_{i}\right)-2-b_{i}(t)>0, \\
& \hat{c}_{i}=4 \sum_{j=1}^{n}\left(\left|c_{i j}(t)\right|+\left|d_{i j}(t)\right|\right) l_{j} .
\end{aligned}
$$

If $\left|y_{i}(t)-y_{i}^{*}\right| \geq 1$, from (4.1), (4.2), and (4.4), we have

$$
V_{i}^{\prime}(t) \leq-\hat{a}_{i}\left(A_{i} x_{i}(t)-A_{i} x_{i}^{*}\right)^{2}-\left(\hat{b}_{i}-\hat{c}_{i}\right)\left(y_{i}(t)-y_{i}^{*}\right)^{2}<0, \quad i \in S
$$

If $\left|y_{i}(t)-y_{i}^{*}\right|<1$, from (4.1), (4.2), and (4.4), we have

$$
V_{i}^{\prime}(t) \leq-\hat{a}_{i}\left(A_{i} x_{i}(t)-A_{i} x_{i}^{*}\right)^{2}-\left(\hat{b}_{i}-\hat{c}_{i}\right)\left|y_{i}(t)-y_{i}^{*}\right|<0, \quad i \in S .
$$

Using conditions (4.1) and (4.2), for any $\varepsilon>0, \iota_{i}-\varepsilon>0$, and $\kappa_{i}-\varepsilon>0$, there exists a positive constant $\mathbb{M}$ (large enough) such that

$$
2 \xi_{i}-1-\left(a_{i}(t)-\xi_{i}\right) \xi_{i}-\frac{b_{i}(t)}{\left.|1-| c_{i}\right|^{2}} \geq \iota_{i}-\varepsilon \quad \text { for } t>\mathbb{M}, i \in S,
$$

and

$$
2\left(a_{i}(t)-\xi_{i}\right)-2-b_{i}(t) \geq \kappa_{i}-\varepsilon \quad \text { for } t>\mathbb{M}, i \in S .
$$

If (4.5) holds, it follows by (4.7) and (4.8) that

$$
V_{i}^{\prime}(t) \leq-\left(\iota_{i}-\varepsilon\right)\left(A_{i} x_{i}(t)-A_{i} x_{i}^{*}\right)^{2}-\left(\kappa_{i}-\epsilon\right)\left(y_{i}(t)-y_{i}^{*}\right)^{2} \quad \text { for } t>\mathbb{M}, i \in S
$$


Take the Lyapunov functional for system (2.3) in the following form:

$$
V(t)=\sum_{i=1}^{n} V_{i}(t), \quad t \in \mathbb{R}
$$

Computing the derivative of it along the solution of system (2.3), and using (4.9), it follows that

$$
V^{\prime}(t) \leq-\sum_{i=1}^{n}\left[\left(l_{i}-\varepsilon\right)\left(x_{i}(t)-x_{i}^{*}\right)^{2}+\left(\kappa_{i}-\varepsilon\right)\left(y_{i}(t)-y_{i}^{*}\right)^{2}\right]<0 \quad \text { for } t>\mathbb{T}, i \in[n]
$$

Integrate both sides of (4.10) from $\mathbb{M}$ to $+\infty$, then

$$
V(t)+\int_{\mathbb{M}}^{+\infty} \sum_{i=1}^{n}\left[\left(\iota_{i}-\varepsilon\right)\left(A_{i} x_{i}(t)-A_{i} x_{i}^{*}\right)^{2}+\left(\kappa_{i}-\varepsilon\right)\left(y_{i}(t)-y_{i}^{*}\right)^{2}\right] \leq V(0) .
$$

By Barbalat's lemma [29], it follows that

$$
\lim _{t \rightarrow+\infty} \sum_{i=1}^{n}\left[\left|A_{i} x_{i}(t)-A_{i} x_{i}^{*}\right|+\left|y_{i}(t)-y_{i}^{*}\right|\right]=0
$$

By Lemma 2.3, we get

$$
\left|x_{i}(t)-x_{i}^{*}\right|=\left|A_{i}^{-1} A_{i}\left(x_{i}(t)-x_{i}^{*}\right)\right| \leq \frac{1}{|1-| c_{i}||}\left|A_{i} x_{i}(t)-A_{i} x_{i}^{*}\right|
$$

which together with (4.11) yields that

$$
\lim _{t \rightarrow+\infty} \sum_{i=1}^{n}\left[\left|x_{i}(t)-x_{i}^{*}\right|+\left|y_{i}(t)-y_{i}^{*}\right|\right]=0 .
$$

If (4.6) holds, we have the same results. The proof of Theorem 4.1 is now finished.

\section{Numerical examples}

This section presents two examples that demonstrate the validity of our theoretical results.

\section{Example 5.1}

$$
\begin{aligned}
\frac{d^{2}\left[A_{1} x_{1}(t)\right]}{d t^{2}}= & -a_{1}(t) \frac{d\left[A_{1} x_{1}(t)\right]}{d t}-b_{1}(t) x_{1}(t)+\sum_{j=1}^{3} c_{1 j}(t) f_{j}\left(x_{j}(t)\right) \\
& +\sum_{j=1}^{3} d_{1 j}(t) f_{j}\left(x_{j}\left(t-\tau_{j}(t)\right)\right)+I_{1}(t), \\
\frac{d^{2}\left[A_{2} x_{2}(t)\right]}{d t^{2}}= & -a_{2}(t) \frac{d\left[A_{2} x_{2}(t)\right]}{d t}-b_{2}(t) x_{2}(t)+\sum_{j=1}^{3} c_{1 j}(t) f_{j}\left(x_{j}(t)\right) \\
& +\sum_{j=1}^{3} d_{2 j}(t) f_{j}\left(x_{j}\left(t-\tau_{j}(t)\right)\right)+I_{2}(t),
\end{aligned}
$$




$$
\begin{aligned}
\frac{d^{2}\left[A_{3} x_{3}(t)\right]}{d t^{2}}= & -a_{3}(t) \frac{d\left[A_{3} x_{3}(t)\right]}{d t}-b_{3}(t) x_{3}(t)+\sum_{j=1}^{3} c_{3 j}(t) f_{j}\left(x_{j}(t)\right) \\
& +\sum_{j=1}^{3} d_{3 j}(t) f_{j}\left(x_{j}\left(t-\tau_{j}(t)\right)\right)+I_{3}(t)
\end{aligned}
$$

where

$$
\begin{aligned}
& T=\frac{\pi}{5}, \quad c_{1}=c_{2}=c_{3}=0.5, \quad a_{1}(t)=a_{2}(t)=a_{3}(t)=0.2, \\
& b_{1}(t)=b_{2}(t)=b_{3}(t)=0.3, \quad c_{i j}(t)=d_{i j}(t)=0.1, \quad \tau_{j}(t)=\frac{1}{2 \pi} \cos 10 t, \\
& f_{j}(u)=\frac{\sin ^{2} u}{u^{2}+1}, \quad I_{1}(t)=I_{2}(t)=I_{3}(t)=\sin 10 t .
\end{aligned}
$$

Obviously, $l_{j}=1,\left|\Gamma_{i j}\right|_{0}=0.211(i, j=1,2,3)$ and Assumption $\left(\mathrm{H}_{2}\right)$ holds. For the above parameters, letting $\xi_{i}=0.1(i=1,2,3)$, we check that conditions (3.5) and (3.6) hold:

$$
\begin{aligned}
& 1-T\left(\left|a_{i}\right|_{0}+\xi_{i}\right)=0.8116>0, \quad i=1,2,3 \\
& 1-\frac{T^{2}|1-| c_{i}||\left(\left|a_{i}\right|_{0}+\xi_{i}\right) \xi_{i}+T^{2}\left|b_{i}\right|_{0}+\left|\Gamma_{i j}\right|_{0}}{|1-| c_{i}||\left(1-T\left(\left|a_{i}\right|_{0}+\xi_{i}\right)\right)\left(1-T \xi_{i}\right)}=0.372>0, \quad i=1,2,3 .
\end{aligned}
$$

Thus, all the assumptions of Theorem 3.2 hold and system (5.1) has at least one $T$-periodic solution. The corresponding numerical simulations are presented in Figs. 1-4 with random initial conditions. Figure 1 shows that system (5.1) possesses at least one $T$-periodic solution. Figures 2-4 show that different subsystems of system (5.1) have periodic solutions.

Example 5.2

$$
\begin{aligned}
\frac{d^{2}\left[A_{1} x_{1}(t)\right]}{d t^{2}}= & -a_{1}(t) \frac{d\left[A_{1} x_{1}(t)\right]}{d t}-b_{1}(t) x_{1}(t)+\sum_{j=1}^{3} c_{1 j}(t) f_{j}\left(x_{j}(t)\right) \\
& +\sum_{j=1}^{3} d_{1 j}(t) f_{j}\left(x_{j}\left(t-\tau_{j}(t)\right)\right)+I_{1}(t)
\end{aligned}
$$

Figure 1 State trajectories of system (5.1) for $(x(t), t)$

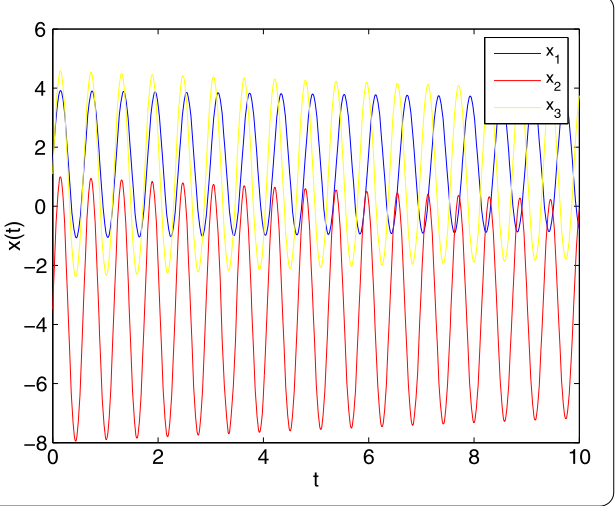


Figure 2 The space diagram of the subsystem $\left(x_{1}(t)\right.$, $\left.x_{2}(t), t\right)$ for system (5.1)

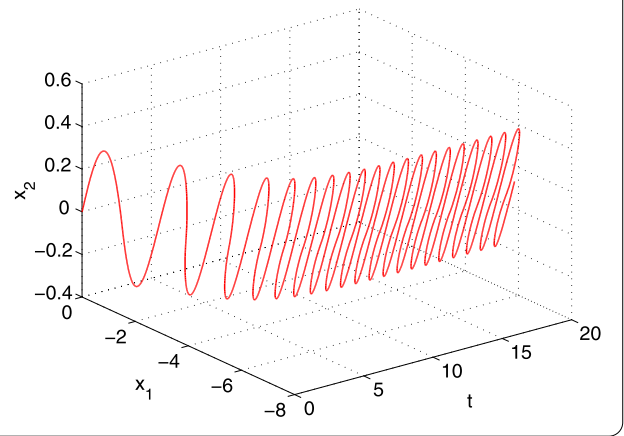

Figure 3 The space diagram of the subsystem $\left(x_{1}(t)\right.$ $\left.x_{3}(t), t\right)$ for system (5.1)

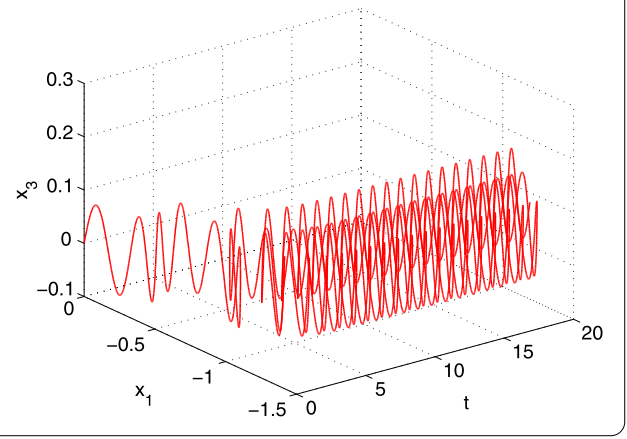

Figure 4 The space diagram of the subsystem $\left(x_{2}(t)\right.$, $\left.x_{3}(t), t\right)$ for system (5.1)

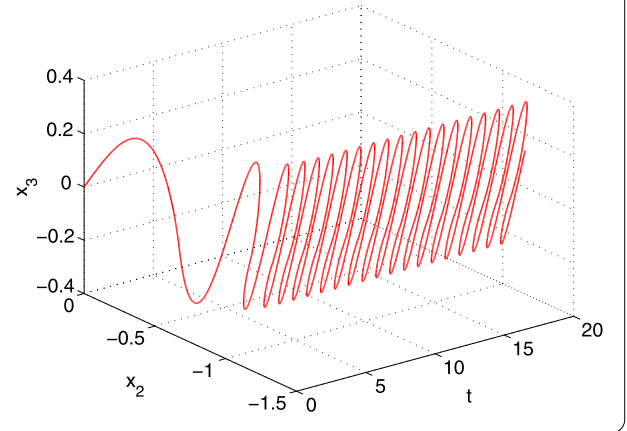

$$
\begin{aligned}
\frac{d^{2}\left[A_{2} x_{2}(t)\right]}{d t^{2}}= & -a_{2}(t) \frac{d\left[A_{2} x_{2}(t)\right]}{d t}-b_{2}(t) x_{2}(t)+\sum_{j=1}^{3} c_{1 j}(t) f_{j}\left(x_{j}(t)\right) \\
& +\sum_{j=1}^{3} d_{2 j}(t) f_{j}\left(x_{j}\left(t-\tau_{j}(t)\right)\right)+I_{2}(t), \\
\frac{d^{2}\left[A_{3} x_{3}(t)\right]}{d t^{2}}= & -a_{3}(t) \frac{d\left[A_{3} x_{3}(t)\right]}{d t}-b_{3}(t) x_{3}(t)+\sum_{j=1}^{3} c_{3 j}(t) f_{j}\left(x_{j}(t)\right) \\
& +\sum_{j=1}^{3} d_{3 j}(t) f_{j}\left(x_{j}\left(t-\tau_{j}(t)\right)\right)+I_{3}(t),
\end{aligned}
$$

where

$$
T=\frac{\pi}{10}, \quad c_{1}=c_{2}=c_{3}=0.9, \quad a_{1}(t)=a_{2}(t)=a_{3}(t)=3,
$$


Figure 5 The periodic solution $x(t)$ of (5.2) is globally asymptotic stable

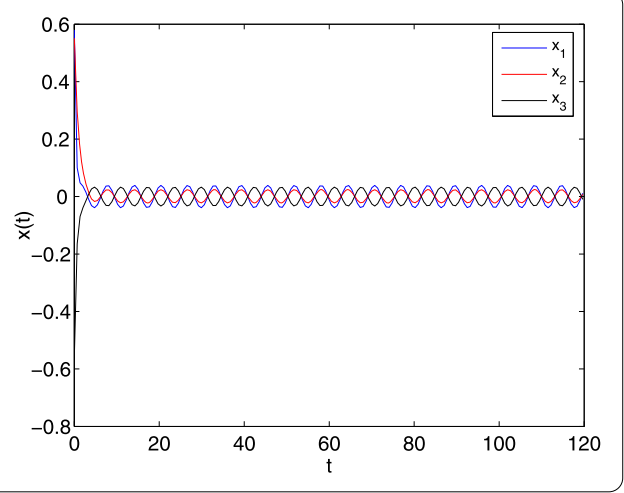

$$
\begin{aligned}
& b_{1}(t)=b_{2}(t)=b_{3}(t)=0.002, \quad c_{i j}(t)=d_{i j}(t)=0.1, \quad \tau_{j}(t)=\frac{1}{2 \pi} \cos 20 t, \\
& f_{j}(u)=\frac{\cos ^{2} u}{u^{2}+1}, \quad I_{i}(t)=\sin t .
\end{aligned}
$$

Obviously $l_{j}=1$. Letting $\xi_{i}=1.8(i=1,2,3)$, we check that conditions (4.1) and (4.2) hold:

$$
\begin{aligned}
\iota_{i} & =\lim _{t \rightarrow+\infty} \inf \left[2 \xi_{i}-1-\left(a_{i}(t)-\xi_{i}\right)^{2} \xi_{i}^{2}-\frac{b_{i}(t)}{\left.|1-| c_{i}\right|^{2}}\right]=1.28>0, \quad i=1,2,3, \\
\kappa_{i} & =\lim _{t \rightarrow+\infty} \inf \left[2\left(a_{i}(t)-\xi_{i}\right)-2-b_{i}(t)-4 \sum_{j=1}^{n}\left(\left|c_{i j}(t)\right|+\left|d_{i j}(t)\right|\right) l_{j}\right]=1.18>0, \\
& i=1,2,3 .
\end{aligned}
$$

Thus, all the assumptions of Theorem 4.1 hold and the periodic solution of (5.2) is globally asymptotically stable. The corresponding numerical simulations are presented in Fig. 5 with random initial conditions. We find that all state orbits of system (5.2) converge to a periodic solution.

Remark 5.1 To the best of our knowledge, the periodic solution problems of neutral-type INNs with delays are considered in the present paper for the first time. Using coincidence degree theory and constructing a proper Lyapunov functional, we got some brand new results on the existence, uniqueness, and asymptotic stability of periodic solutions of neutral-type INNs. We can confirm the novelty of the proposed methods: for example, the methods in [18,30-33] cannot be generalized to the problems studied in this article. It is important to point out that global exponential stability results of an equilibrium in Lagrange sense for neutral-type INNs were obtained by Theorem 3.1 in [19], and in this paper we only obtain some sufficient conditions for global asymptotic stability of a periodic solution of neutral-type INNs, we have not solved the problem of global exponential stability. The main reason is that constructing a proper Lyapunov functional is very difficult in a periodic function space. We hope to study the global exponential stability of the periodic solution of system (2.1) in a future research.

Remark 5.2 Using Matlab for ODE, we give Figs. 1-5 which show the properties of systems (5.1) and (5.2). Figure 1 shows that system (5.1) has a periodic solution. Figures 2-4 
show phase diagrams for system (5.1) in three different states $\left(x_{1}, x_{2}, t\right),\left(x_{1}, x_{3}, t\right),\left(x_{2}, x_{3}, t\right)$. Figure 5 shows that system (5.2) has a periodic solution which is stable.

\title{
6 Conclusions and discussions
}

In this paper we studied the problems of periodic solutions for neutral-type inertial neural networks with multiple variable delays. First, by applying Mawhin's continuous theorem to the system, we got a set of sufficient conditions for the existence and uniqueness of periodic solutions. Then, on the basis of existence results, we obtained global asymptotic stability of periodic solutions. The efficacy of the obtained results has been demonstrated by two numerical examples. It is important to note that the practical implementation of INNs is typically encountered with certain type of uncertainties such as interval parameters. Extending the results of this paper to neutral-type INNs with interval uncertainties proves to be an interesting problem. In addition, it is also interesting and challenging to extend the approach presented in this paper to neural network-based problems with mixed delays such as state estimation and approximation, fault isolation and diagnosis, or filter/observer design. These issues require further investigations in the future works.

Since exponential stability implies asymptotic stability, the exponential stability problem of neutral-type inertial neural networks is more important than the asymptotic stability problem of neutral-type inertial neural networks. In this paper, we only obtained some asymptotic stability results for neutral-type inertial neural networks, and we hope that some exponential stability results for neutral-type inertial neural networks will be obtained in the future.

\author{
Acknowledgements \\ The authors would like to thank the editor and the referees for their valuable comments and suggestions that improved \\ the quality of our paper. \\ Funding \\ The work is supported by Natural Science Foundation of Jiangsu High Education Institutions of China (Grant No. \\ 17KJB110001) \\ Availability of data and materials \\ Data sharing not applicable to this article as no datasets were generated or analyzed during the current study. \\ Competing interests \\ The authors declare that they have no competing interests. \\ Authors' contributions \\ All authors contributed equally to the writing of this paper. All authors read and approved the final manuscript.
}

\section{Publisher's Note}

Springer Nature remains neutral with regard to jurisdictional claims in published maps and institutional affiliations.

Received: 30 June 2020 Accepted: 19 October 2020 Published online: 29 October 2020

\section{References}

1. Wheeler, D., Schieve, W.: Stability and chaos in an inertial two-neuron system. Phys. D: Nonlinear Phenom. 105, 267-284 (1997)

2. Koch, C:: Cable theory in neurons with active linearized membrane. Biol. Cybern. 50, 15-33 (1984)

3. Babcock, K., Westervelt, R.: Stability and dynamics of simple electronic neural networks with added inertia. Phys. D: Nonlinear Phenom. 23, 464-469 (1986)

4. Tu, Z., Cao, J., Hayat, T.: Matrix measure based dissipativity analysis for inertial delayed uncertain neural networks. Neural Netw. 75, 47-55 (2016)

5. Wan, P., Jian, J.: Global convergence analysis of impulsive inertial neural networks with time-varying delays. Neurocomputing 245, 68-76 (2017)

6. Tu, Z., Cao, J., Hayat, T.: Global exponential stability in Lagrange sense for inertial neural networks with time-varying delays. Neurocomputing 171, 524-531 (2016) 
7. Wang, J., Tian, L.: Global Lagrange stability for inertial neural networks with mixed time-varying delays. Neurocomputing 235, 140-146 (2017)

8. Arik, S.: Global robust stability analysis of neural networks with discrete time delays. Chaos Solitons Fractals 26, 1407-1414 (2005)

9. Zhou, T., Liu, Y., Liu, Y.: Existence and global exponential stability of periodic solution for discrete-time BAM neural networks. Appl. Math. Comput. 182, 1341-1354 (2006)

10. Zhou, T., Liu, Y., Li, X., Liu, Y.: Global exponential periodicity for BAM neural network with periodic coefficients and continuously distributed delays. Comput. Math. Appl. 55, 2689-2698 (2008)

11. Liu, B., Huang, L.: Existence and exponential stability of periodic solutions for a class of Cohen-Grossberg neural networks with time-varying delays. Chaos Solitons Fractals 32, 617-627 (2007)

12. Yin, H., Du, B., Yang, Q., Duan, F.: Existence of homoclinic orbits for a singular differential equation involving p-Laplacian. J. Funct. Spaces 2020, 1-7 (2020)

13. Zhang, Z., Peng, G., Zhou, D.: Periodic solution to Cohen-Grossberg BAM neural networks with delay on time scales. J. Franklin Inst. 348, 2754-2781 (2011)

14. Zhou, T., Du, B., Du, H.: Positive periodic solution for indefinite singular Liénard equation with $p$-Laplacian. Adv. Differ. Equ. 2019, 158 (2019)

15. Du, B: Anti-periodic solutions problem for inertial competitive neutral-type neural networks via Wirtinger inequality. J. Inequal. Appl. 2019, 187 (2019)

16. Lu, W., Chen, T.: $\mathbb{R}_{+}^{n}$-global stability of a Cohen-Grossberg neural network system with nonnegative equilibria. Neural Netw. 20, 714-722 (2007)

17. Ding, H., Liu, Q., Nieto, J.: Existence of positive almost periodic solutions to a class of hematopoiesis model. Appl. Math. Model. 40, 3289-3297 (2016)

18. Hien, L., Hai-An, L.: Positive solutions and exponential stability of positive equilibrium of inertial neural networks with multiple time-varying delays. Neural Comput. Appl. 31, 6933-6943 (2019)

19. Yogambigai, J., Syed Ali, M., Alsulami, H., Alhodaly, M.S.: Global Lagrange stability for neutral-type inertial neural networks with discrete and distributed time delays. Chin. J. Phys. 65, 513-525 (2020)

20. Hale, J.: The Theory of Functional Differential Equations. Springer, New York (1977)

21. Yin, H., Du, B.: Stochastic patch structure Nicholson's blowfies system with mixed delays. Adv. Differ. Equ. 2020, 386 (2020)

22. Park, J., Park, C.H., Kwon, O.M., Lee, S.M.: A new stability criterion for bidirectional associative memory neural networks of neutral-type. Appl. Math. Comput. 199(2), 716-722 (2008)

23. Park, J., Kwon, O.M., Lee, S.M.: LMI optimization approach on stability for delayed neural networks of neutral-type. Appl. Math. Comput. 196(1), 236-244 (2008)

24. Yu, K.W., Lien, C.H.: Stability criteria for uncertain neutral systems with interval time-varying delays. Chaos Solitons Fractals 38(3), 650-657 (2008)

25. Wang, B., Liu, X., Zhong, S.: New stability analysis for uncertain neutral system with time-varying delay. Appl. Math Comput. 197(1), 457-465 (2008)

26. Gaines, R., Mawhin, J.: Coincidence Degree and Nonlinear Differential Equations. Springer, Berlin (1977)

27. $L u, S:$ On the existence of positive periodic solutions for neutral functional differential equation with multiple deviating arguments. J. Math. Anal. Appl. 280, 321-333 (2003)

28. Lu, S., Renee, J., Ge, W.: Problems of periodic solutions for a kind of second order neutral functional differential equation. Appl. Anal. 82, 411-426 (2003)

29. Barbalat, I.: Systems d'equations differential d'oscillationsn onlinearities. Rev. Rounmaine Math. Pure Appl. 4, 267-270 (1959)

30. Li, Y.K.: Existence and global attractivity of a positive periodic solution of a class of delay differential equation. Sci. China Ser. A 41(3), 273-284 (1998)

31. Chen, W., Liu, B.: Positive almost periodic solution for a class of Nicholson's blowflies model with multiple time-varying delays. J. Comput. Appl. Math. 235, 2090-2097 (2011)

32. Ding, H., Alzabut, J.: Existence of positive almost periodic solutions for a Nicholson's blowflies model. Electron. J. Differ. Equ. 2015, 180 (2015)

33. Hien, L., Hai-An, L.: Positive solutions and exponential stability of positive equilibrium of inertial neural networks with multiple time-varying delays. Neural Comput. Appl. 31, 6933-6943 (2019)

\section{Submit your manuscript to a SpringerOpen ${ }^{\circ}$ journal and benefit from:}

- Convenient online submission

- Rigorous peer review

- Open access: articles freely available online

- High visibility within the field

- Retaining the copyright to your article

Submit your next manuscript at $\gg$ springeropen.com 